Artigo

\title{
La formación estadística de los profesores de matemáticas. Caso Venezuela.
}

The statistical training of math teachers. Venezuela case.

A formação estatística de professores de matemática. Caso Venezuela.

Audy Salcedo ${ }^{1}$
$[0000-0002-9783-8509]$

\section{Resumo}

O objetivo desta pesquisa é analisar a proposta de formação estatística para professores de matemática na Venezuela. A utilização da estatística no quotidiano e a sua utilidade nas diferentes áreas do conhecimento tem levado ao reconhecimento do seu valor para a educação geral de todos os cidadãos. Por esta razão, já há várias décadas, a maioria dos países incluiu estatísticas como parte do currículo de matemática do ensino fundamental e médio. Em qualquer mudança curricular, o papel do professor é essencial para o seu sucesso. Mas as instituições de formação também são importantes, que devem fazer ajustes nos currículos para formar as novas gerações de professores para ensinar os novos conteúdos. Por isso, este trabalho relata a análise dos planos de estudos para a formação de professores de matemática de duas universidades venezuelanas, com vistas a estabelecer como eles aspiram a prepará-los para o ensino de estatística e probabilidade. Os resultados indicam que a proposta de formação não parece adequada para que os futuros professores lecionem estatística no ensino médio.

Palavras-chave: Formação de professores. Formação estatística do cidadão. Currículo de matemática. Competência matemática.

\begin{abstract}
The objective of this research is to analyze the statistical training proposal for mathematics teachers in Venezuela. The use of statistics in everyday life and its usefulness in different areas of knowledge has led to the recognition of its value for the general education of all citizens. For this reason, for several decades now, most countries have included statistics as part of the primary and secondary mathematics curriculum. In any curricular change, the role of the teacher is essential for its success. However, training institutions are also important, which must make adjustments in the curricula to train new generations of teachers to teach the new content. That is why this work reports the analysis of the study plans for the training of mathematics teachers from two Venezuelan universities, with a view to establishing how they aspire to prepare them for the teaching of statistics and probability. The results indicate that the training proposal does not seem to be adequate for future teachers to teach statistics in secondary school.
\end{abstract}

Keywords: Teacher training. Citizen statistical training. Mathematics curriculum. Mathematical competence.

1 audy.salcedo@gmx.com, Doctor en Educación, Profesor, Universidad Central de Venezuela / Universidad Católica del Maule, Talca/Maule/Chile. 


\section{Resumen}

El objetivo de esta investigación es analizar la propuesta de formación estadística para profesores de matemáticas de Venezuela. El uso de la estadística en la vida cotidiana y su utilidad en distintas áreas de conocimiento ha llevado al reconocimiento de su valor para la formación general de todo ciudadano. Por ello, desde hace ya varias décadas, la mayoría de los países han incluido a la estadística como parte del currículo de matemáticas de primaria y secundaria. En cualquier cambio curricular el papel que desempeña el profesor es esencial para su éxito. Pero también son importantes las instituciones de formación, las cuales deben hacer ajustes en los planes de estudios para capacitar a las nuevas generaciones de docentes en enseñanza de los nuevos contenidos. Es por ello que en este trabajo se reporta el análisis de los planes de estudio para la formación de profesores de matemáticas de dos universidades venezolanas, con miras a establecer cómo se aspira a prepararlos para la enseñanza de la estadística y la probabilidad. Los resultados indican que la propuesta de formación no parece ser adecuada para que los futuros profesores enseñen estadística en secundaria.

Palabras claves: Formación de profesores. Formación estadística del ciudadano. Currículo de matemáticas. Competencia matemática.

\section{Introducción}

La utilidad de la estadística en una amplia variedad de áreas de conocimiento, así como su uso frecuente en la vida cotidiana, han llevado a la mayoría de los países a incorporar a la estadística como parte de la educación general que recibe todo ciudadano. La recomendación es que se enseñe desde los primeros grados de la educación básica (BEN-ZVI, MAKAR, 2016; National Council of Teachers of Mathematics [NCTM], 2006), ya que se desea que el ciudadano alcance la alfabetización estadística (Asociación Americana de Estadística [ASA], 2016; Crites y St. Laurent, 2015). Son tres las razones por la que la estadística y la probabilidad deben incluirse en la formación del ciudadano: (a) son esenciales para el razonamiento crítico; (b) tienen un rol instrumental en otras disciplinas, y (c) son fundamentales para la planificación y la toma de decisiones en muchas profesiones (BATANERO; BOROVCNIK, 2016).

Además, la estadística permite una mejor comprensión de los problemas políticos en la sociedad moderna. Para que las sociedades democráticas funcionen, los ciudadanos deben conocer y comprender críticamente la evidencia cuantitativa sobre cuestiones clave relacionadas con el bienestar social, económico, sanitario y el logro de los derechos civiles. En consecuencia, las de habilidades estadísticas son necesarias para la participación informada (ENGEL; RIDGWAY; WEBER, 2021).

En la mayoría de los países la estadística se encuentra en el currículo de matemática de primaria y secundaria, por lo cual maestros y profesores son los responsables de impartir de los contenidos de probabilidad y estadística. En el caso de Venezuela, la estadística está en la educación secundaria desde la década de los 70 . Fue parte de las modificaciones al currículo producto de las recomendaciones emanadas de la Segunda Conferencia Interamericana de Educación Matemática, donde se sugería que los jóvenes de 15 a 18 años estudiaran temas de Probabilidad y Estadística Elemental (BARRANTES, RUIZ, 1998). Con la reforma curricular efectuada en los años 80 , el estudio de la estadística se inicia en el primer grado de educación primaria y de probabilidad desde el quinto.

Al referirse a la incorporación de la estadística en los programas de matemáticas de la Educación Básica, Becerra y Moya (1989) señalan que su presencia desde el primer grado es una de las principales innovaciones de esa reforma curricular. Explican que la estadística es un 
instrumento fundamental para el desarrollo científico y tecnológico. A finales de los 90, se produce un nuevo cambio curricular en la educación básica venezolana, centrado en el desarrollo de competencias. Los programas de matemáticas se modifican, se mantienen los contenidos de estadística desde primer grado, pero la probabilidad se comienza a estudiar en segundo (MINISTERIO DE EDUCACIÓN, 1997).

Toda reforma trae consigo retos y dificultades en su instrumentación y siempre hay que considerar el importante rol que juegan los docentes para su éxito. En 1966, Luis Santaló, representante de Argentina en la Segunda Conferencia Interamericana de Educación Matemática, advertía sobre la importancia de considerar la preparación de los profesores para llevar adelante la enseñanza con los nuevos programas. Por lo que proponía la actualización de los docentes en servicio e introducir cambios en los planes de los institutos dedicados a la formación de profesores con el propósito de que salieran preparados para llevar a cabo los programas que proponía la reforma (BARRANTES; RUIZ, 1998).

Considerando que la estadística está en los programas de matemáticas de Venezuela desde hace, por lo menos, 50 años, es de suponer que no es necesaria la actualización de los docentes activos. Las instituciones de formación docente han tenido suficiente tiempo para hacer ajustes en los planes de estudios y capacitar a las nuevas generaciones de docentes para enseñanza de la estadística y probabilidad.

Aunque en la revisión bibliográfica realizada se encontraron algunos trabajos sobre la formación de profesores de matemáticas en Venezuela (LEÓN, 2016; BEYER; LEÓN; IGLESIAS; SERRES, 2017), no se localizó ninguna investigación que abordara el tema de su formación estadística. A nivel internacional, ha aumentado la investigación sobre el conocimiento estocástico de los profesores, no obstante, el tema de su formación estadística es aún prioritario y uno de los aspectos a considerar es el análisis de las propuestas instruccionales, en sus diferentes facetas (BATANERO, 2019). Es por ello que esta investigación tiene por objetivo analizar la propuesta de formación para la enseñanza y el aprendizaje de la estadística y la probabilidad que ofrecen las instituciones de formación para profesores de matemáticas en Venezuela. En particular se analizan los planes de estudio de dos de las universidades venezolanas de mayor peso en la formación de docentes.

\section{Marco de referencia}

\subsection{Necesidad de la alfabetización estadística de los profesores}

Para Gal (2004) la alfabetización estadística es una de las competencias que deben desarrollar todos los ciudadanos y está vinculada a la posibilidad de evaluar e interpretar críticamente la información estadística. Indica

que se refiere a dos componentes interrelacionados: a) capacidad para interpretar y evaluar críticamente la información estadística, los argumentos apoyados en datos o los fenómenos estocásticos que las personas pueden encontrar en diversos contextos, incluyendo los medios de comunicación, pero no limitándose a ellos, y b) capacidad para discutir o comunicar sus opiniones respecto a tales informaciones estadísticas cuando sea relevante (GAL, 2002, pp. 2-3).

Eso significa que un ciudadano puede ser considerado alfabetizado estadísticamente cuando cuenta con una base de conocimientos que le permita organizar y comunicar de 
manera eficiente la información recolectada en diferentes contextos, además de poder comunicar y argumentar sobre las conclusiones alcanzadas. Para Gal (2004), "la alfabetización estadística [...] es necesaria para que los adultos sean plenamente conscientes de las tendencias y fenómenos de importancia social y personal" (p. 49).

Otros investigadores (por ejemplo, BATANERO; GODINO, 2005; WATSON, 2001; BATANERO; BOROVCNIK, 2016) también consideran que la alfabetización estadística es parte importante de la formación general de todo ciudadano y por ello apoyan la inclusión de la estadística desde los primeros años de la educación primaria.

Batanero (2019) señala "hoy día personas con poca formación en estocástica deben usar estas ideas enfrentado a los medios de comunicación o en el trabajo profesional en situaciones que requieren razonamiento estocástico para su correcta interpretación" (p. 11). La investigación indica que los docentes, responsables de la enseñanza de la estadística y la probabilidad, también demuestran dificultades respecto a la compresión las ideas estadísticas fundamentales. Por ejemplo, Estrella et al. (2015), Molina-Portillo et al. (2018), Fernández et al. (2019), Cavalcanti y Guimarães (2019). Souza y Monteiro (2020), Salcedo et al. (2020), reportan dificultades por parte de docentes (activos o en formación) en la comprensión e interpretación de gráficos estadísticos.

Rodríguez-Alveal y Díaz-Levicoy (2020) reportan que futuros profesores no se consideran competentes para resolver problemas asociados a probabilidad básica y no están interesados en enseñar probabilidad a sus futuros estudiantes. Estos investigadores señalan que, al enfrentarse a situaciones para la toma de decisiones, los estudiantes de su muestra no declaran elementos teóricos referentes a modelos estadísticos y su argumentación carece de fundamentos teóricos, por lo que señalan que esos estudiantes no han adquirido un razonamiento probabilístico.

López-Martin et al. (2019) evaluaron los conocimientos de un grupo de futuros profesores españoles de Educación Secundaria y Bachillerato, con relación a los errores de sus estudiantes en la inferencia estadística. Encontraron que, aunque son capaces de identificar errores, los describen con poca precisión, lo cual sugiere que su conocimiento del tema es todavía escaso; por ejemplo, cuando se alude a hipótesis mal planteadas, a la interpretación incorrecta del intervalo de confianza.

Las dificultades de los docentes (activos o en formación) en la compresión las ideas estadísticas no son recientes. Por ejemplo, al hacer un balance de esas dificultades identificadas en la investigación, Batanero et al. (2011) señalan que tienen poca comprensión de las medidas de tendencia central, dificultades para crear o interpretar gráficos; problemas en la compresión de la variación, poca comprensión de la desviación estándar como una medida de la homogeneidad de la muestra; confusión entre correlación y causalidad; dificultades para diferenciar una prueba de hipótesis estadística de una prueba matemática. Todos esos aspectos se refirieren a problemas que tienen los profesores sobre el contenido estadístico que necesitan para enseñar, pero también suelen tener problemas respecto a cómo enseñarlo, ya que con mucha frecuencia ponen el énfasis en procedimientos algorítmicos y no en la compresión de las ideas estadística fundamentales. La compresión de contenido a enseñar y el cómo enseñarlo son aspectos que deben ser alcanzados en las instituciones donde se forman los docentes, de ahí la importancia del tema de este trabajo. 


\subsection{Conocimiento matemático para la enseñanza}

Shulman (1986) planteo la pregunta ¿de dónde provienen las explicaciones del profesor? Esa y otras preguntas lo llevan a replantearse la premisa según la cual el conocimiento del profesor se fundamentaba en dos dominios independientes: el Conocimiento del Contenidos (Content knowledge) y el Conocimiento Pedagógico (Pedagogical Knowledge). Propone un tercer constructo, superposición de los dos anteriores: Conocimiento Pedagógico del Contenidos (Pedagogical Content knowledge, PCK). Señala que en él incluye "los tópicos más regularmente enseñados en un área de contenido, las formas más útiles de representación de estas ideas; las analogías, ilustraciones, ejemplos, explicaciones y demostraciones más poderosos; en pocas palabras, las formas de representación y formulación del tema que lo hace comprensible a otros" (SHULMAN, 1986, p. 9). Pero también considera otros conocimientos, aquellos que son producto de su experiencia y reflexión del docente, como "la compresión de lo que hace fácil o difícil el aprendizaje de tópicos específicos: las concepciones y preconcepciones que los estudiantes de diferentes edades y características traen cuando aprenden los tópicos más frecuentemente enseñados" (SHULMAN, 1986, p. 9).

Posteriormente, Shulman (1987) señala el PCK es una "mezcla entre el contenido y la pedagogía en un esfuerzo por comprender cómo un tópico, problema o tema específico es organizado, representado y adaptado para los diversos intereses y habilidades de los estudiantes y presentado para la enseñanza" (p. 8). Amplía y profundiza en las categorías del conocimiento base que un profesor necesita para enseñar un determinado contenido, considerando como mínimo las siguientes: (a) conocimiento del contenido; (b) conocimiento pedagógico general, con énfasis en los principios generales y estrategias de gestión de aula y organización; (c) conocimiento del currículo, especialmente lo referido a la comprensión, de materiales y programas que sirven como "herramientas del oficio" para los profesores; (d) conocimiento pedagógico del contenido; (e) conocimiento de los estudiantes y sus características; (f) conocimiento de los contextos educativos, que va desde el trabajo del grupo o clase, hasta la administración y financiamiento escolar en distintas comunidades y culturas; (g) conocimiento de los fines, propósitos y valores de la educación, así como de sus fundamentos históricos y filosóficos (SHULMAN, 1987, p. 8).

Los trabajos de Shulman llamaron la atención de los investigadores del área de formación docente, pero pronto observaron que el PCK no es un constructo único e igual para todas las áreas, ni para todos los docentes de un área temática determinada. En el caso de la formación del profesor de matemáticas, un grupo de investigadores (BALL, 2000; BALL et al., 2001; HILL et al., 2008) proponen el Conocimiento Matemático para la Enseñanza (Mathematical Knowledge for Teaching, MKT).

En Ball et al. (2008) se define el MKT "conocimiento matemático necesario para realizar las tareas recurrentes de enseñar matemáticas a los estudiantes" (p. 399). En esa línea Hill et al. (2008) propone un modelo de conocimiento matemático para la enseñanza, el cual se estructura sobre la base de dos grandes componentes, el conocimiento del contenido y el conocimiento pedagógico del contenido. El primero es un conocimiento propio del profesor de matemáticas, parte de lo que lo define como profesional, su conocimiento sobre la matemática. se fundamenta en:

- Conocimiento común del contenido. Es "aquel conocimiento que es usado en el trabajo de enseñanza en formas comunes a como se utiliza en muchas otras 
profesiones u ocupaciones que también usan matemáticas" (HILL et al., 2008, p. 377).

- Conocimiento del horizonte matemático. Conocimiento de relaciones entre los conocimientos matemáticos escolares y el contexto curricular.

- Conocimiento especializado del contenido. Es el "conglomerado de conocimientos y habilidades matemáticas exclusivas para la enseñanza" (BALL et al., 2008, p. 400).

El segundo subdominio, conocimiento pedagógico del contenido, es el conocimiento matemático para la enseñanza y va más allá del conocimiento matemático y pedagógico general, incluye los conocimientos de conceptos e ideas matemáticas para y en la enseñanza de la matemática. Está formado por:

- Conocimiento del contenido y los estudiantes. Es el conocimiento sobre "cuál es la mejor manera de construir el pensamiento matemático en los estudiantes, o cómo solucionar los errores de los estudiantes. Está focalizado en la comprensión de los profesores de cómo los estudiantes aprenden un contenido particular" (HILL et al., 2008, p. 378).

- Conocimiento del contenido y la enseñanza. Es el conocimiento de las metodologías adecuadas para llevar a cabo el proceso de enseñanza y aprendizaje de un determinado tema.

- Conocimiento del currículo. Se refiere a las características del currículo que el profesor debe conocer para así articular, de mejor manera, los distintos contenidos con otras asignaturas o dentro de su misma asignatura.

Otros investigadores (CARRILLO et al., 2013; FLORES et al., 2013; MONTES et al., 2013) redefinen el modelo del Conocimiento Matemático para la Enseñanza y proponen el Modelo del Conocimiento Especializado del Profesor de Matemáticas (Mathematics Teacher's Specialized Knowledge, MTSK). Este nuevo modelo conserva los dos grandes tipos de conocimiento, pero en lugar de hablar del Conocimiento Especializado del Contenido lo renombran como el Conocimiento Matemático. Además, este agrega las creencias respecto a ambos tipos de conocimientos: a la matemática y la enseñanza y el aprendizaje de la matemática.

Los componentes del Conocimiento Matemático son:

- Conocimiento de los temas. "Este subdominio se concreta en saber matemáticas; conocer los aspectos fenomenológicos asociados al tema; conocer los distintos significados del tema; conocer ejemplos específicos en aspectos concretos de un tema matemático, etc." (ROJAS et al., 2015, p. 147)

- Conocimiento de la estructura matemática. Considera una visión de conjunto de la matemática, la idea de conexión y complejidad del contenido matemático. Incluye los vínculos entre ideas o conceptos matemáticos distintos, así como los conocimientos previos y posteriores con el contenido de enseñanza (ROJAS et al., 2015, p. 147)

- Conocimiento de la práctica de la matemática. Se refiere a los conocimientos sobre los modos de proceder de las matemáticas formales. Sus formas de conocer, producir conocimiento y comunicarlos (ROJAS et al., 2015, p. 148).

El conocimiento didáctico del contenido matemático también incluye tres componentes:

- Conocimiento de la enseñanza de las matemáticas. Incluye el conocimiento de teorías de enseñanza de la matemática, pero también los recursos didácticos que permitan al estudiante adquirir, reforzar o ejercitar los contenidos (ROJAS et al, 2015, p. 148). 
- Conocimiento de las características del aprendizaje de las matemáticas. Es el "conocimiento que permite al profesor conocer el modo de pensar de los estudiantes sobre las tareas matemáticas y comprende todo lo relacionado con generar aprendizaje de las matemáticas" (ROJAS et al, 2015, p. 148).

- Conocimiento de los estándares de aprendizaje. Se refiere a los conocimientos sobre los contenidos propuestos en currículo oficial, pero también considera las recomendaciones de expertos, asociaciones profesionales o de investigadores, sin dejar de lado la propia experiencia (ROJAS et al., 2015, p. 148).

Los modelos de Conocimiento Matemático para la Enseñanza y Conocimiento Especializado del Profesor de Matemáticas tienen como raíz común los trabajo de Shulman y tienen más coincidencias que diferencias. Por ejemplo, aunque usan denominaciones distintas, las dimensiones conocimiento pedagógico del contenido y conocimiento didáctico del contenido matemático puede considerarse equivalentes. La principal diferencia es la incorporación de las creencias acerca de las matemáticas y su enseñanza en el Modelo Conocimiento Especializado del Profesor de Matemáticas. Ambos modelos comparten una concepción de un profesional de la docencia centrado en las matemáticas institucionalizadas por la escuela, por lo que pueden ser apropiados para analizar los planes de formación de profesores de matemáticas.

\section{Metodología}

Este trabajo busca a establecer cómo se aspira a formar a los profesores de matemática de Venezuela para la enseñanza de la estadística y la probabilidad. Para ello se analizan los planes de estudio para la formación de profesores de matemáticas de dos universidades: Universidad Pedagógica Experimental Libertador y Universidad Nacional Abierta. Se hace un análisis desde la perspectiva de los programas de estudio oficiales, en consecuencia, es una investigación documental sobre la base del currículo escrito (POSNER, 2005).

La Universidad Pedagógica Experimental Libertador (UPEL), es la universidad formadora de docentes por excelencia de Venezuela. Está conformada por ocho instituciones regionales, las cuales abarcan una gran porción de Venezuela. Esta institución cubre la mayor demanda de formación de docentes de educación secundaria del país en las distintas especialidades. Por su parte, la Universidad Nacional Abierta (UNA) la institución de educación superior a distancia más antigua de Venezuela, que cuenta con un programa de formación de profesores de matemáticas a nivel nacional.

En la UNA, la carrera está prevista cursarla en 10 semestres ( 5 años) y recibe el título de Licenciado en Educación Mención Matemática, mientras que en la UPEL el plan de estudio está distribuido 8 semestres (4 años) y al graduarse recibe el título de Profesor de Matemáticas.

En la estructura de ambas carreras se aprecia la influencia de los trabajos de Shulman, ya que clasifican las asignaturas en:

- Componente de formación general.

- Componente de formación pedagógica.

- Componente de formación especializada.

- Prácticas profesionales.

Inicialmente se respetó esa clasificación, aunque luego trató por separado las asignaturas de lo que Rojas et al. (2015) denominan el Conocimiento Matemático y el 
Conocimiento Didáctico del Contenido Matemático, dos de los aspectos que Batanero (2009) destaca entre los retos de la formación estadística de profesores. Al tratarlos por separado se puede tener una mejor idea de la propuesta de formación que se encuentra en los planes de estudios de las instituciones consideradas.

\section{Resultados}

A continuación, se presentan los datos recopilados de los planes de estudio para la formación de profesores de matemáticas de dos universidades: Universidad Pedagógica Experimental Libertador y Universidad Nacional Abierta. En el cuadro 1 se muestra las asignaturas de ambos planes.

Cuadro 1 - Asignaturas en los componentes de la formación de profesor de matemática.

\begin{tabular}{|c|c|c|c|c|}
\hline \multirow[b]{2}{*}{ Componente } & \multicolumn{2}{|c|}{ UPEL } & \multicolumn{2}{|c|}{ UNA } \\
\hline & $\begin{array}{c}\text { Número } \\
\text { Asignaturas }\end{array}$ & $\%$ & $\begin{array}{c}\text { Número } \\
\text { Asignaturas }\end{array}$ & $\%$ \\
\hline General & 15 & 28,3 & 8 & 22,2 \\
\hline Pedagógico & 10 & 18,9 & 4 & 11,1 \\
\hline Especializado & 16 & 30,2 & 16 & 44,4 \\
\hline Educación matemática & 8 & 15,1 & 6 & 16,7 \\
\hline Práctica profesional & 4 & 7,5 & 2 & 5,6 \\
\hline Total & 53 & 100 & 36 & 100 \\
\hline
\end{tabular}

Fuente: Elaboración propia

En ambas universidades el mayor número de asignaturas corresponden al Componente Especializado, las asignaturas vinculadas con el Conocimiento Matemático, según Rojas et al. (2015). Aunque tienen igual número de asignaturas (16), en la UPEL representa un porcentaje menor, ya que esta universidad tiene un plan de estudios con más asignaturas, a pesar de tener un año menos de duración. En esta institución el Componente General y Pedagógico aportan algo más del $47 \%$ a la formación del profesor, mientras el Componente Especializado es algo más del $30 \%$.

La UPEL es donde hay un mayor número de asignaturas asociadas al componente de Educación Matemática o Conocimiento Didáctico del Contenido Matemático (Rojas, et al., 2015). La proporción de asignaturas vinculadas a la Educación Matemática ocupa la 4ta posición en la UPEL y el 3er lugar en la UNA, de nuevo la explicación está en el número de asignaturas de la UPEL, ya que la diferencia en valor absoluto es de 2 asignaturas a favor de la UPEL. En ambas universidades, la Práctica Profesional son los de menor aporte porcentual a la formación del profesor de matemática. Esto significa un menor contacto con lo que posteriormente será su ámbito de trabajo.

Para valorar la formación estadística del profesor es necesario examinar con más detalle el Conocimiento Matemático y el Conocimiento Didáctico del Contenido Matemático (educación matemática) de cada institución. En el cuadro 2 presentan las asignaturas que conforman el Conocimiento Matemático.

Cuadro 2 - Asignaturas del Conocimiento Matemático de la formación de profesor de matemática. 


\begin{tabular}{|c|c|c|c|c|}
\hline \multirow[b]{2}{*}{ Área } & \multicolumn{2}{|c|}{ UPEL } & \multicolumn{2}{|c|}{ UNA } \\
\hline & $\begin{array}{c}\text { Número } \\
\text { Asignaturas }\end{array}$ & $\%$ & $\begin{array}{c}\text { Número } \\
\text { Asignaturas }\end{array}$ & $\%$ \\
\hline Álgebra & 4 & 25,0 & 2 & 12,5 \\
\hline Cálculo y Análisis & 5 & 31,3 & 6 & 37,5 \\
\hline Geometría & 3 & 18,8 & 1 & 6,3 \\
\hline Estadística y Probabilidad & 1 & 6,3 & 2 & 12,5 \\
\hline Otras asignaturas & 3 & 18,8 & 5 & 31,3 \\
\hline Total & 16 & 100 & 16 & 100 \\
\hline
\end{tabular}

Fuente: Elaboración propia.

En el cuadro 2, se parecía que las áreas de tradición en la matemática, Álgebra y Cálculos y Análisis, son las que tienen un mayor número de asignaturas y en consecuencia un mayor peso porcentual en el conocimiento matemático del futuro profesor de matemáticas egresado de estas instituciones. En la distribución de las asignaturas hay un claro desequilibrio en contra de las referidas al pensamiento estocástico y a favor de uso de modelos matemáticos determinísticos.

La UPEL tiene tres asignaturas que se vincularon al Conocimiento Matemático, aunque no se trata de las áreas propiamente matemáticas: (a) Historia de la matemática, (b) Rigor y formalización en matemática y (c) Matemática y ciencias naturales. Las tres podrían ubicarse en el subdominio Conocimiento de la Estructura de las Matemáticas (Rojas et al., 2015), ya aportan en la formación del futuro profesor de matemáticas en cuanto al proceder de las matemáticas formales y a las conexiones intra matemáticas y extra matemáticas. Es importante acotar que estas tres asignaturas son electivas y no necesariamente un futuro profesor las tendría que cursar. En las UNA, las asignaturas ubicadas en esa categoría son: (a) Presentación a la Física, (b) Física I, (c) Física II, (d) Tópicos de Matemática y (e) Matemáticas y Ciencias; las cuales también se consideran del subdominio Conocimiento de la Estructura de las Matemáticas. Todas son asignaturas obligatorias.

En cuanto al número de asignaturas el área de Estadística y Probabilidad, así como la de Geometría, podrían tener un impacto menor en la formación de profesores. Esas son dos de los temas de matemáticas que tradicionalmente se reportan como menos trabajadas por los profesores en sus clases de secundaria.

En el caso de la UNA, son dos cursos, uno de probabilidad y otro de estadística, mientras que en la UPEL es un único curso. En todos los casos se trata de asignaturas con enfoques exclusivamente matemáticos con poca o ninguna vinculación con las aplicaciones de la estadística o con su didáctica. Por ejemplo, el curso de Estadística y Probabilidad de la UPEL se declara que tiene como propósito "proveer al estudiante y futuro docente de matemática de conocimientos y habilidades en el manejo de conceptos, principios y técnicas de la estadística descriptiva y de las probabilidades" (UPEL, 2015, p. 107). Ese propósito se refleja en la lista de contenidos:

Conceptos básicos de Estadística. Fuentes de información. Técnicas e instrumentos de recolección de datos estadísticos. Organización y presentación de datos estadísticos. Medidas de posición y de tendencia central. Medidas de variabilidad y de forma. Análisis de Correlación Lineal 
simple. Métodos de conteo, principios básicos. Incertidumbre. Probabilidad. Concepciones. Axiomas y Leyes de probabilidad. Variables aleatorias. Distribuciones de probabilidad. (UPEL, 2015, p. 108)

La primera parte del curso es de estadística descriptiva, luego se encuentra el tópico de correlación lineal simple y al final aparecen algunos fundamentos de probabilidad. Esto deja fuera de la formación de estos futuros docentes todo lo relativo a la inferencia estadística. Eso es una desventaja en su formación como profesional y ciudadano, ya que podría tener problemas al momento de, por ejemplo, leer artículos de investigación o de prensa que incluyan procesos de inferencia. Difícilmente, un estudiante que curse solo está asignatura pueda lograr la alfabetización estadística por la que aboga Gal (2002).

En el caso de la UNA, el curso de Probabilidad el objetivo es: "Aplicar con destreza los conceptos y teoremas fundamentales de la teoría de la probabilidad" (UNA, 2014, p. 7). Los contenidos del curso son:

Elementos de combinatoria. Variaciones, permutaciones y combinaciones. Fórmula de Steaffel. Fórmula del binomio de Newton. Espacio muestral. Evento. Muestreo con reposición y sin reposición. Espacio de probabilidad y sus consecuencias. Criterio de estimación por máxima verosimilitud. Probabilidad uniforme. Definiciones y resultados básicos sobre probabilidad condicional. Teorema de Bayes. Definición de independencia. Eventos independientes. Aplicaciones. Definición de variable aleatoria y operaciones con variables aleatorias. Función de distribución de variables aleatorias discretas: Poisson, Binomial e Hipergeométrica. Función de distribución y densidades de variables aleatorias continuas: Uniforme, Triangular, Exponencial, Normal. Distribución conjunta de dos variables aleatorias. Distribuciones marginales. Variables aleatorias independientes. Distribución conjunta de variables aleatorias independientes. Distribución conjunta de variables aleatorias discretas. Densidades conjuntas. Distribución Multinomial. Suma de variables aleatorias independientes. Caso discreto y caso absolutamente continuo. Funciones de variables aleatorias independientes. Definición y propiedades básicas de la esperanza matemática. Caso discreto y continuo. Momentos de orden superior. Ley fuerte de los grandes números. Desigualdad de Markov. Desigualdad de Tchebichev. Lema de Borel- Cantelli. Teorema Central del Límite. Método de Montecarlo. (UNA, 2014, pp. 7 y 8 )

Se parte de los conceptos básicos de la teoría combinatoria, para luego pasar a los fundamentos de la probabilidad y profundizar con las variables aleatorias y las distribuciones de probabilidad. Culmina con Desigualdad de Tchebichev y el Teorema Central del Límite, con lo cual se dan los pasos iniciales hacia la inferencia estadística. Se trata de un curso de probabilidad, que de acuerdo al objetivo declarado debería dirigirse a las aplicaciones.

La asignatura Estadística de la UNA declara como objetivo "Analizar situaciones concretas que admitan el tratamiento con métodos y cálculos estadísticos en el campo de la educación, administración y la industria" (UNA, 2018, p. 7). Para poder cursar esta asignatura, los estudiantes deben aprobar previamente el curso de Probabilidad, descrito antes. Los contenidos del curso son: 
Muestreo aleatorio y probabilidades. Ley de los grandes números. Teorema del lımite central. Estadísticos muestrales. Función de distribución empírica. Histogramas y ojivas. Distribuciones Chi-cuadrado, T de Student, distribución F y sus respectivas tablas. Distribuciones gamma y exponencial. Distribución de la media y diferencia de medias en poblaciones normales. Independencia de la media muestral y de la varianza muestral. Varianza combinada y simplificación de la diferencia entre dos medias. Razón de varianzas. Planteamiento del problema de estimación. Estimación puntual. Propiedades de los estimadores. Estimados insesgado de mínima varianza. Estimador de los momentos y sus propiedades. Estimador de máxima verosimilitud y sus propiedades. Intervalos de confianza. Método para construir intervalos de confianza. Intervalos de confianza para medias y varianzas. Intervalos de confianza para el parámetro $\mathrm{p}$ de la distribución binomial. Hipótesis' estadística. Contraste de hipótesis. Tipos de errores. Elección del contraste. Contraste de hipótesis simples y compuesta para la media, varianza y diferencia de medias. Uso del p-valor. Contraste $\chi 2$. Prueba de bondad de ajuste. Tablas de contingencia. Pruebas de independencia. Modelo de regresión. Estimación de los coeficientes de regresión. Coeficiente de determinación. Correlación entre dos o más variables. Contraste de hipótesis para elegir un modelo. Análisis de varianza. Modelo de clasificación simple y estimación de parámetros. Modelo de clasificación simple de efecto aleatorio. Modelo de clasificación doble y estimación de los parámetros. Modelo de clasificación doble de efecto aleatorio. (UNA, 2018, p. 5)

Un detalle importante de las asignaturas de la UNA, es que son comunes para estudiantes de ingeniería y educación matemática, lo cual lo podría hacer interesante en cuanto a la variedad de aplicaciones de la probabilidad y la estadística que se discutan en el curso. Pero al mismo tiempo es una desventaja si se pretenden discutir problemas más cercanos a la educación matemática. Que las asignaturas de Probabilidad y Estadística de la UNA estén inclinadas hacia las aplicaciones podría favorecer lo que Rojas et al. (2015) denominan el Conocimiento de la Estructura Matemática.

Batanero et al. (2011) señala que el conocimiento estadístico de los docentes juega un papel significativo en la calidad de su enseñanza, ya que las decisiones instruccionales que debe tomar dependen de ese conocimiento. De acuerdo a lo analizado, el profesor egresado de a UPEL podría tener dificultades para la enseñanza de la estadística, ya que su formación en el contenido se remite a unos pocos aspectos de estadística descriptiva y probabilidad. Pero también, significa menos formación para comprender y evaluar la información estadística a la cual está expuesto como ciudadano. En el cuadro 3 presenta las asignaturas incluidas en el Conocimiento Didáctico del Contenido Matemático, de ambas instituciones.

Cuadro 3 - Asignaturas incluidas en el Conocimiento didáctico del contenido matemático de la formación de profesor de matemática.

\begin{tabular}{|ll|ll|}
\hline \multicolumn{1}{|c|}{ UPEL } & \multicolumn{1}{c|}{ UNA } \\
\hline - & Matemática y Educación Especial & $\bullet$ & Didáctica de la Aritmética \\
- & Problemática de la Enseñanza de la Matemática & $\bullet$ & Teoría de la Educación Matemática \\
- Didáctica del Algebra & $\bullet$ & Didáctica del Álgebra y la Trigonometría \\
- Didáctica de la Matemática & $\bullet$ & Evaluación de los Aprendizajes en Matemática \\
\hline
\end{tabular}


- Matemática y Realidad

- Didáctica del Cálculo

- El Juego y la Creatividad

- Resolución de Problemas
- Didáctica de la Geometría

- Didáctica de la Estocástica

Fuente: Elaboración propia.

De las ocho asignaturas de la UPEL incluidas en el componente de Conocimiento Didáctico del Contenido Matemático, solo Didáctica de la Matemática es obligatoria, todas las demás forman parte del conjunto de posibles electivas que el futuro docente puede decidir cuál cursar. Dos de ellas se enfocan en la didáctica de temas específicos de la matemática (Calculo y Algebra), mientras que las demás abordan teorías o recursos en la enseñanza de la matemática, por lo se consideran de la sub dimensión Conocimiento de la enseñanza de las matemáticas. Ninguna de esas electivas tiene relación con la enseñanza o el aprendizaje de la estadística y la probabilidad,

Aunque la asignatura Didáctica de la Matemática tiene como propósito:

el desarrollo de habilidades y destrezas para el análisis y la solución de problemas propios del proceso de enseñanza y aprendizaje de la Matemática mediante la elaboración, revisión o ensayo de secuencias de enseñanza diseñadas sobre la base de proposiciones didácticas fundamentadas relativas a dicho proceso, con el fin de garantizar su desempeño eficiente como facilitador del aprendizaje de la Matemática en los niveles y modalidades del sistema educativo en los cuales le corresponda actuar. (UPEL, 2015, pp. 118 y 119)

En los contenidos a estudiar no encuentran puntos específicos vinculados a enseñanza o el aprendizaje de la estadística y la probabilidad, como se puede apreciar en el listado siguiente:

Problemática de la enseñanza y el aprendizaje de la Matemática en Venezuela. Conceptualización de la Educación Matemática, la investigación, áreas y líneas de Investigación en Educación Matemática. Naturaleza y Estructura de la Matemática: Nociones básicas relativas a la historia, filosofía y naturaleza de la Matemática. Rasgos esenciales y metodológicos del conocimiento matemático. Estructura actual y significación sociocultural de la Matemática. Fines de la enseñanza de la Matemática. Teorías contemporáneas de la instrucción y del aprendizaje, aplicables a la enseñanza de la Matemática. Proposiciones didácticas para la enseñanza y el aprendizaje de la Matemática factibles de ser aplicadas en Venezuela. (UPEL, 2015, p. 119)

Tanto el propósito como la lista de contenidos parecen corresponder a una asignatura de teoría de la didáctica de la matemática o teoría de la educación matemática, propia de la sub dimensión Conocimiento de la Enseñanza de las Matemáticas, sin considerar aspectos de las otras dos sub dimensiones del modelo de Rojas et al. (2015). La inclusión de temas de específicos de enseñanza o aprendizaje de la estadística o probabilidad (así como de cualquier otro tema) dependerá de la profundidad y orientación que desee darle el profesor de la asignatura.

Dos detalles a destacar con respecto a la asignatura de Didáctica de la Matemática de la UPEL. Primero, para cursarla es necesario haber aprobado la asignatura Estadística y 
Probabilidad. En teoría, eso significaría que los conocimientos logrados en Estadística y Probabilidad son necesarios para cursar Didáctica de la Matemática. Pero ese requisito parece artificial al no haber contenidos específicos de estadística o probabilidad en esa didáctica. El otro detalle es que la bibliografía de la asignatura solo incluye tres fuentes, dos referidas a la enseñanza de la geometría y una a la matemática en general, lo cual parece ratificar la desvinculación de esa asignatura con la enseñanza y aprendizaje con la estadística y la probabilidad.

En la UNA, las seis asignaturas relacionadas con el Conocimiento didáctico del contenido matemático y también se pueden ubicar la sub dimensión Conocimiento de la Enseñanza de las Matemáticas, ya que en ellas se estudian teorías sobre la enseñanza de la matemática, así como recursos didácticos. A diferencia de la UPEL, la UNA si tiene una asignatura específica para trabajar aspectos del aprendizaje y la enseñanza de la estadística y la probabilidad.

Didáctica de la Estocástica tiene como objetivo: "Aplicar de forma crítica, los entornos de aprendizaje de la estadística, la probabilidad tomando en cuenta los objetivos de la enseñanza, el nivel de desarrollo de los estudiantes y el contexto social cultural de los mismos" (UNA, 2006, p. 4). Los contenidos de esa asignatura son:

Teorías e investigación en la enseñanza, aprendizaje y evaluación de la estocástica. Problemática de la educación estocástica. Concepciones alternas de los estudiantes en la estocástica. Creencias e interpretaciones de la probabilidad y la estadística. Errores de los estudiantes. Aleatoriedad. Cultura, razonamiento y pensamiento estadístico. Discusión de investigaciones recientes. La probabilidad y la estadística del entorno y de la actividad humana: aplicaciones académicas, tecnológicas y cotidianas de la estocástica. Diversos conceptos de probabilidad. Medición y Predicción. Proyectos para la enseñanza de la estocástica. La estocástica en los programas oficiales en Venezuela. Contenidos básicos de la estadística y la probabilidad. Estándares NCTM, currículo nacional inglés, estándares colombianos y otros programas. Integración de la estadística y la probabilidad con otros contenidos matemáticos. Materiales curriculares en estocástica, tipos y características. Recursos en Internet. Fuentes de datos. Software especializado para la enseñanza de la estadística y la probabilidad. Simulaciones. Integración de la estadística y la probabilidad con otros contenidos matemáticos. Diseño y criterios para el análisis de entornos de aprendizaje para la enseñanza de la estadística y la probabilidad con diversas tecnologías. Proyectos integradores. Actividades extracurriculares en estadística y probabilidad. Prácticas Escolares. (UNA, 2006, p. 4)

Aunque esta asignatura se considera de la sub dimensión Conocimiento de la Enseñanza de las Matemáticas, el listado de contenidos permite apreciar que abordaran aspectos de Conocimiento de las características del aprendizaje de las matemáticas al abordar, por ejemplo, las concepciones alternas de los estudiantes, sus creencias e interpretaciones de la probabilidad y la estadística, así como los errores más frecuentes. También incluiría aspectos del Conocimiento de los estándares de aprendizaje, al considerar los programas oficiales de Venezuela y de otros países.

Aunque la UPEL tiene un mayor número de asignaturas vinculadas al Conocimiento Didáctico del Contenido Matemático, la propuesta de la UNA, al incluir la asignatura Didáctica 
de la Estocástica, considera los aspectos particulares de la enseñanza y el aprendizaje de la estadística y la probabilidad, a donde no es posible trasladar los principios generales de otros temas de la matemática. Así como la Estadística creció como un campo aparte de la Matemática, la Educación Estadística se ha desarrollado como una disciplina distinta de la Educación Matemática, con problemas de investigación propios (ZIEFFLER et al., 2018). Es por ello que la formación de profesores de matemáticas debe incorporar esos conocimientos particulares de la enseñanza y el aprendizaje de la estadística y la probabilidad.

\section{A manera de cierre}

Las asignaturas de los planes de estudio de las instituciones de formación de profesores de matemática analizadas en este trabajo, se pueden agrupar en dos bloques: (a) un componente teórico y (b) otro práctico. El componente teórico se puede subdividir en cuatro grupos: las de formación general, las de aspectos generales de educación, las referidas al conocimiento matemático y las relacionadas con la enseñanza de la matemática. Las prácticas profesionales son el otro bloque de asignaturas, donde el estudiante aplica la teoría aprendida previamente. La perspectiva epistemológica que subyace en esos planes de estudio es la parcelación de los saberes y la desvinculación de la teoría con la práctica. Es lo que Porlán y Rivero (1998), denominan el enfoque tecnológico, según la cual el docente es la suma de conocimientos referidos al contenido, conocimientos sobre educación, algunas técnicas de enseñanza. La formación en estadística del profesor de matemáticas venezolano no escapa de ese esquema.

En cuanto al Conocimiento Matemático, es claro el desbalance a favor de las asignaturas de corte determinístico respecto a las vinculadas al pensamiento estocástico en ambas instituciones analizadas. Que durante la formación de los profesores se haga más énfasis en asignaturas de modelos matemáticos determinísticos, implícitamente le confieren un mayor nivel de importancia, en desmedro de la estadística y la probabilidad. En consecuencia, los profesores podrían presidir de esos temas cuando en momentos de apremio deban sacrificar algún contenido, como fue el caso de la pandemia del COVID19.

La formación en el contenido estadístico de los futuros egresados de la UPEL es limitada, no los favorece ni como profesionales ni como ciudadanos que necesita apoyarse en la estadística en su vida cotidiana. En el caso de la UNA, de acuerdo con los programas analizados, favorece las aplicaciones en el mundo actual y abona un mejor terreno para el desarrollo de su alfabetización estadística. No obstante, pareciera adecuada ampliar esa formación en el contenido estadístico.

Con relación al componente de Conocimiento Didáctico del Contenido Matemático, también la UNA tiene una mejor propuesta en la formación estadística del profesor de matemáticas al incluir en su plan de estudios la asignatura Didáctica de la Estocástica; mientras que la UPEL no aborda temas de la enseñanza y aprendizaje de la estadística de la probabilidad, ni en la asignatura Didáctica de la Matemática ni en las electivas que ofrece a sus estudiantes.

Pareciera que los futuros docentes egresados de las instituciones analizadas, recibirían una escueta formación para la enseñanza y el aprendizaje de la estadística y la probabilidad en la educación secundaria venezolana. Esto sin duda influye en su futuro desempeño como docentes y en el valor que le concedan a la estadística en el marco de los programas de matemática. Los resultados de esta investigación sugieren que las instituciones de formación 
docente podrían tener una deuda de más de 50 años con la formación estadística de los profesores de matemáticas. Por ello se cree necesario introducir cambios en las instituciones de formación de docentes para mejorar la preparación en estadística y su conocimiento didáctico de los futuros docentes.

Es importante destacar que los principios generales del conocimiento didáctico del contenido de otras áreas de la matemática no pueden ser transferidos a la estadística, así mismo, el razonamiento estadístico no es similar a otras áreas de la matemática, por lo que hace falta una formación específica para enseñar estadística. También es importante ensayar otro esquema de formación como el sugerido por Ball (2000) o Rojas et al. (2015), quienes proponen hacer, de manera simultánea, la enseñanza de los contenidos matemáticos con sus aspectos didácticos, de tal forma que se transforme en el modelo a seguir en su desempeño profesional.

\section{Agradecimiento}

El autor agradece el apoyo del Institute of International Education's Scholar Rescue Fund (IIE-SRF) para su estancia en el Centro de Investigación en Educación Matemática y Estadística de la Facultad de Ciencias Básicas de la Universidad Católica del Maule.

\section{Referencias}

BALL, Deborah L. Bridging practices: Intertwining content and pedagogy in teaching and learning to teach. Journal of Teacher Education, v. 51, p. 241-247, 2000.

BALL, Deborah L.; LUBIENSKI, Sarah. T.; MEWBORN, Denise. S. Research on teaching mathematics: The unsolved problem of teachers' mathematical knowledge. En V. Richardson (Ed.), Handbook of research on teaching (4th ed., pp. 433-456). 2001. Washington, DC: American Educational Research Association.

BALL, Deborah L.; THAMES, Mark H.; PHELPS, Geoffrey. Content knowledge for teaching: What makes it so special? Journal of Teacher Education, v. 59, p. 389-407, 2008.

BARRANTES, Hugo; RUIZ, Ángel. Historia del Comité Interamericano de Educación Matemática. Bogotá: Academia Colombiana de Ciencias Exactas, Físicas y Naturales. 1998

BATANERO, Carmen. Retos para la formación estadística de los profesores. II Encontro de Probabilidade e Estatística na Scola. Universidade do Minho, 2009, Braga, Portugal. https://www.ugr.es/ batanero/pages/ARTICULOS/Formprofesores.pdf

BATANERO, Carmen. Treinta años de investigación en educación estocástica: Reflexiones y desafíos. In J. M. Contreras, M. M. Gea, M. M. López-Martín y E. Molina-Portillo (Eds.), Actas del Tercer Congreso Internacional Virtual de Educación Estadística. 2019. www.ugr.es/local/fqm126/civeest.html

BATANERO, Carmen; BOROVCNIK, Manfred. Statistics and probability in high school. Rotterdamn, The Netherlands: Sense Publishers. 2016. 
BATANERO, Carmen; GODINO, Juan D. Perspectivas de la educación estadística como área de investigación. In: R. Luengo (Ed.) Líneas de investigación en Didáctica de las Matemáticas. Badajoz: Universidad de Extremadura, p. 203-226, 2005.

https://www.ugr.es/ batanero/pages/ARTICULOS/Perspectivas.pdf

BATANERO, Carmen; BURRILL, Gail; READING, Chris. Overview: challenges for teaching statistics in school mathematics and preparing mathematics teachers. In: BATANERO, Carmen, BURRILL, Gail; READING, Chris (Eds.), Teaching Statistics in School-Mathematics-Challenges for Teaching and Teacher Education: A Joint ICMI/IASE Study. Springer, p. 407- 418, 2011. https://doi.org/10.1007/978-94-007-1131-0.

BECERRA, Rosa.; MOYA, Andrés. La matemática como una perspectiva innovadora y fundamental en el nivel de Educación Básica. In Oficina Sectorial de Planificación y Presupuesto (Edit.). Ponencias y Trabajos Libres I Congreso Nacional de Educación Caracas: Ministerio de Educación, v. 207 - 216, 1989.

BEN-ZVI, Dani; MAKAR, Katie. International perspectives on the teaching and learning of statistics. In: BEN-ZVI, Dani; MAKAR, Katie (Eds.), The Teaching and Learning of Statistics. International Perspectives. New York, NY: Springer, p. 1-10, 2016.

BEYER, Walter; LEÓN, Nelly; IGLESIAS, Martha; SERRES, Yolanda. La formación del docente de matemática en Venezuela. Matua. Revista del Programa de Matemáticas, v. IV, p. 56-66. 2017.

CARRILLO, José; CLIMENT, Eric; CONTRERAS, Luis; MUÑOZ-CATALÁN, María del Carmen. Determining Specialized Knowledge for Mathematics teaching. In: UBUZ, Behiye, HASER, Çiğdem \& MARIOTTI, Maria A. Proceedings of the Eighth Congress of the European Society for Research in Mathematics Education. Antalya, Turkey: CERME, p. 2985 - 2994, 2013.

CAVALCANTI, Milka; GUIMARÃES, Gilda. Conhecimentos de professores dos anos iniciais (regular e EJA) sobre escala representada em gráficos. In: CONTRERAS, José M., GEA, Maria M., LÓPEZ-MARTÍN; María M.; MOLINA-PORTILLO, Elena (Eds.), Actas del Tercer Congreso Internacional Virtual de Educación Estadística, 2019. doi: https://www.ugr.es/ fqm126/civeest/cavalcanti.pdf

CRITES, Terry; ST. LAURENT, Roy. Putting essential understanding of statistics into practice in grades 9-12. Reston, VA: National Council of Teachers of Mathematics. 2015

ENGEL, Joachim; RIDGWAY, Jim; WEBER, Stein, Florian. Educación Estadística, Democracia y Empoderamiento de los Ciudadanos. Paradigma, v. 41, n.e1, p. 01-31. 2021. https://doi.org/10.37618/PARADIGMA.1011-2251.2021.p01-31.id1016

ESTRELLA, Soledad, OLFOS, Raimundo \& MENA-LORCA, Arturo. El conocimiento pedagógico del contenido de estadística en profesores de primaria. Educação e Pesquisa, v. 41, n.2, p. 477-493. 2015. https://doi.org/10.1590/s1517-97022015041858

FLORES, Eric; ESCUDERO, Dinazar I.; CARRILLO, José. A Theoretical Review of Specialised Content Knowledge. En: UBUZ, Behiye, HASER, Çiğdem; MARIOTTI, Maria A. (Eds.), Proceedings of the Eighth Congress of the European Society for Research in Mathematics Education. Antalya, Turkey: CERME, p. $3055-3064,2013$. 
GAL, Iddo. Adult's statistical literacy. Meanings, components, responsibilities. International Statistical Review, v.70, n.1, p.1-25. 2002

GAL, Iddo. Statistical Literacy: Meanings, Components, Responsibilities. In: BEN-ZIV, Dani \& GARFIELD, Joan (Ed). The Challenge of Developing Statistical Literacy. Dordrecht: Kluwer Academic Publishers, p. 47-78, 2004.

HILL, Heather C., BLUNK, Merrie L., CHARALAMBOUS, Charalambos Y., LEWIS, Jennifer M., PHELPS, Geoffrey, SLEEP, Laurie; BALL, Deborah L. Mathemarical knowledge for teaching and the mathematical quality of instruction an exploratory study. Cognition and Instruction, v.26, n.4, p. 430$511,2008$.

LEÓN, Nelly. La formación del docente de Matemática en Venezuela: una acción en tiempo progresivo. Cuadernos de Investigación y Formación en Educación Matemática. n.15, p. 443-457, 2016.

LÓPEZ-MARTíN, María del Mar, BATANERO, Carmen; GEA, María M. ¿Conocen los futuros profesores los errores de sus estudiantes en la inferencia estadística? Bolema: Boletim de Educação Matemática, v.33, n.64, p. 672-693, 2019. https://doi.org/10.1590/1980-4415v33n64a11

MINISTERIO DE EDUCACIÓN. Currículo Básico Nacional. Programa de Estudio de Educación Básica. Caracas: Autor. 1997.

MOLINA-PORTILLO, Elena, CONTRERAS, José Miguel, RUZ, Felipe; CONTRERAS, Javier. Evaluación de la cultura estadística en futuros profesores de educación primaria: Interpretación y argumentación de gráficos estadísticos. En: RODRÍGUEZ-MUÑIZ, Luis J., MUÑIZ RODRÍGUEZ, Laura, AGUILARGONZÁLEZ, Alvaro, ALONSO, Pedro, GARCÍA GARCíA Francisco J.; BRUNO, Alicia (Eds.), Investigación en Educación Matemática XXII, Gijón: SEIEM, p. 348-357, 2018.

MONTES, Miguel; AGUILAR, Álvaro; CARRILLO, José; MUÑOZ-CATALÁN, M. Cinta. MTSK: From Common and Horizon Knowledge to Knowledge of Topics and Structures. En: UBUZ, Behiye, HASER, Çiğdem \& MARIOTTI, Maria A. Proceedings of the Eighth Congress of the European Society for Research in Mathematics Education, Antalya, Turkey: CERME. Antalya, Turquía, p. 3185-3194. 2013.

NCTM (2006). Curriculum focal points for prekindergarten through grade 8 mathematics. Reston, VA: Autor.

PORLÁN, Rafael; RIVERO, Ana. El conocimiento de los profesores. Una propuesta formativa en el área de ciencias. Sevilla. España: Díada. 1998.

POSNER, George J. Análisis del currículo. Bogotá: McGraw-Hill. 2005

ROJAS, Nielka; FLORES, Pablo; CARRILLO, José. Conocimiento Especializado de un Profesor de Matemáticas de Educación Primaria al Enseñar los Números Racionales. Boletim de Educação Matemática, v.29, n.51, p.143-166, 2015. https://doi.org/10.1590/1980-4415v29n51a08

SALCEDO, Audy, GONZÁLEZ, Jesús, SARCO LIRA, Amalio \& GONZÁLEZ, Johnnalid. Maestros en formación leen e interpretan gráficos estadísticos Jornal Internacional de Estudos em Educação Matemática. v.13, n.4, 2020. https://revista.pgsskroton.com/index.php/jieem/article/view/7985 
SHULMAN, Lee S. Knowledge and Teaching: Foundations of the New Reform. Harvard Educational Review, v.57, n.1, p.1-23, 1987. https://doi.org/10.17763/haer.57.1.j463w79r56455411

SHULMAN, Lee S. Those who understand: Knowledge growth in teaching. Educational Research, v. 15, n. 2, p. 4-14. 1986. https://doi.org/10.3102/0013189x015002004

SOUZA, Josilane de \& MONTEIRO, Carlos E. F. Compreensões sobre gráficos por professores de escolas no campo. Zetetike, v.28, e020005. 2020. https://doi.org/10.20396/zet.v28i0.8657061

UNA. Plan de curso. Didáctica de la Estocástica (577). Mimeografiado. 2006

UNA. Plan de curso. Introducción a la Probabilidad (737). Probabilidad (747). Mimeografiado. 2014

UNA. Plan de curso. Inferencia Estadística (738) Estadística (748). Mimeografiado. 2018

UPEL. Diseño curricular de matemática. Autor. 2015

WATSON, Jane M. Profiling teacher's competence and confidence to teach particular mathematics topics: The case of chance and data. Journal of Mathematics Teacher Education, v. 4, n.4, p. 305337. 2001.

ZIEFFLER, Andrew, GARFIELD, Joan; FRY, Elizabeth. What Is Statistics Education? In: BEN-ZVI, Dani, MAKAR, Katie \& GARFIELD, Joan (eds) International Handbook of Research in Statistics Education. Springer International Handbooks of Education. Springer, Cham. 2018. https://doi.org/10.1007/9783-319-66195-7_2 